\title{
Radiological diagnosis of hepatocellular carcinoma in non-cirrhotic patients
}

\author{
Alba Castán', Ylenia Navarro1, Luis Sarría1, Raquel Larrosa1, Mario Serradilla², Alejandro Serrablo² \\ ${ }^{I}$ Department of Radiology, Miguel Servet University Hospital, 50009 Zaragoza, Spain \\ ${ }^{2}$ Division of Hepato-Pancreato-Biliary, Department of Surgery, Miguel Servet University Hospital, 50009 Zaragoza, Spain.
}

Correspondence to: Dr. Luis Sarría, Department of Radiology, Miguel Servet University Hospital, Paseo Isabel la Católica, 1-3, 50009 Zaragoza, Spain. E-mail: 1sarriao@salud.aragon.es

How to cite this article: Castán A, Navarro Y, Sarría L, Larrosa R, Serradilla M, Serrablo A. Radiological diagnosis of hepatocellular carcinoma in non-cirrhotic patients. Hepatoma Res 2017;3:1-17.

\author{
Article history: \\ Received: 05-11-2015 \\ Accepted: 17-10-2016 \\ Published: 12-01-2017 \\ Key words: \\ Hepatocellular carcinoma, \\ non-cirrhotic liver, \\ ultrasound, \\ elastography, \\ computed tomography, \\ magnetic resonance
}

\begin{abstract}
\end{abstract}
\section{INTRODUCTION}

Primary liver cancer is the fifth most common cancer in men and the ninth most common cancer in women, assuming the second leading cause of cancer death worldwide. ${ }^{[1]}$ Eighty-three percent of new cases occur in developing countries, half of them in China. Its incidence has increased in recent decades, especially in developed countries. In 2015 in Spain, the incidence was 5.172 cases per 100,000 population and there was an emergence of about 32,000 new cases in the United States. ${ }^{[1]}$

Up to $90 \%$ of primary liver tumors are hepatocellular carcinoma (HCC). HCC has its origin in hepatocytes, the predominant cells of the liver parenchyma. Around $80-90 \%$ arises in a cirrhotic liver. The most commonly associated risk factors are chronic infection with hepatitis B virus (HBV) and hepatitis C virus $(\mathrm{HCV})$. HBV is the most common cause of $\mathrm{HCC}$

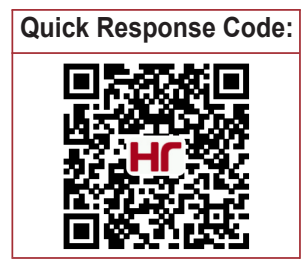


in underdeveloped countries. In developed countries, most HCC originate in a setting of alcoholic cirrhosis or non-alcoholic steatosis related to obesity. However, there is an incidence of $0.5-1 \%$ per year in patients with non-cirrhotic livers. ${ }^{[2]}$ Usually, such patients are not subject to monitoring prevention programs and so HCC detection is usually late and secondary to symptoms produced by the tumor. Less frequent risk factors are type II diabetes and metabolic syndrome, congenital diseases such as hereditary hemochromatosis, tobacco, parasitic infections or genotoxin intake. The average age at diagnosis of $\mathrm{HCC}$ is 63 years old, with an incidence three times higher in men than in women. ${ }^{[2]}$

Clinically, it is a silent disease in early stages. When symptoms appear, the most common is abdominal pain $(52 \%) .{ }^{[3]}$ Less common symptoms are chronic diarrhea, jaundice, fever, or paraneoplastic syndromes such as hypercalcemia or hypoglycemia. It may occur with increased serum levels of alpha-fetoprotein, considered indicative of HCC above $400 \mathrm{ng} / \mathrm{dL} .{ }^{[4]}$ However, this determination has low sensitivity and specificity for diagnosis and for monitoring.

\section{RADIOLOGICAL DIAGNOSIS OF HCC}

There are three basic diagnostic tests: computed tomography (CT), magnetic resonance imaging (MRI) and ultrasound (US).

\section{Computed tomography}

Proper technique is essential for the accurate assessment of HCC: a baseline study, an arterial phase after administration of intravenous contrast (30-35 s), a portal phase (75-90 s) and a late phase (after $3 \mathrm{~min}$ ). HCC presents as a single nodular lesion in most cases. Around $20 \%$ are multinodular. Without contrast, its density is similar to normal or slightly lower than liver parenchyma. Contrast series shows a typical dynamic behavior. It is a tumor with neoangiogenesis of arterial origin; therefore, it enhances intensely in arterial phase. In portal phase (venous) and late phase, the tumor washes the contrast and becomes hypodense relative to normal parenchyma [Figure 1].

This behavior of early enhancement and late washing (wash in - wash out) is part of the main diagnostic criteria for HCC. Its mosaic appearance is also characteristic with areas of different density within the liver, visible especially in post-contrast phases. The tumor is often encapsulated, identifying one hypodense halo. The capsule enhances more slowly and gradually and uptake usually persists in later stages. Sometimes, the edges are imprecise, which also determines more aggressive tumors. Growth is usually expansive although there may be transcapsular infiltration into the surrounding parenchyma.

However, a high percentage of patients do not demonstrate pathognomonic HCC criteria, showing atypical features. Thus, in a retrospective study of 243 patients conducted by Lee et al., ${ }^{[5]}$ the most typical behavior of tumors corresponded to moderately differentiated HCC. A high percentage of cases showed atypical behavior (43.6\%). Most of these tumors corresponded histologically to well
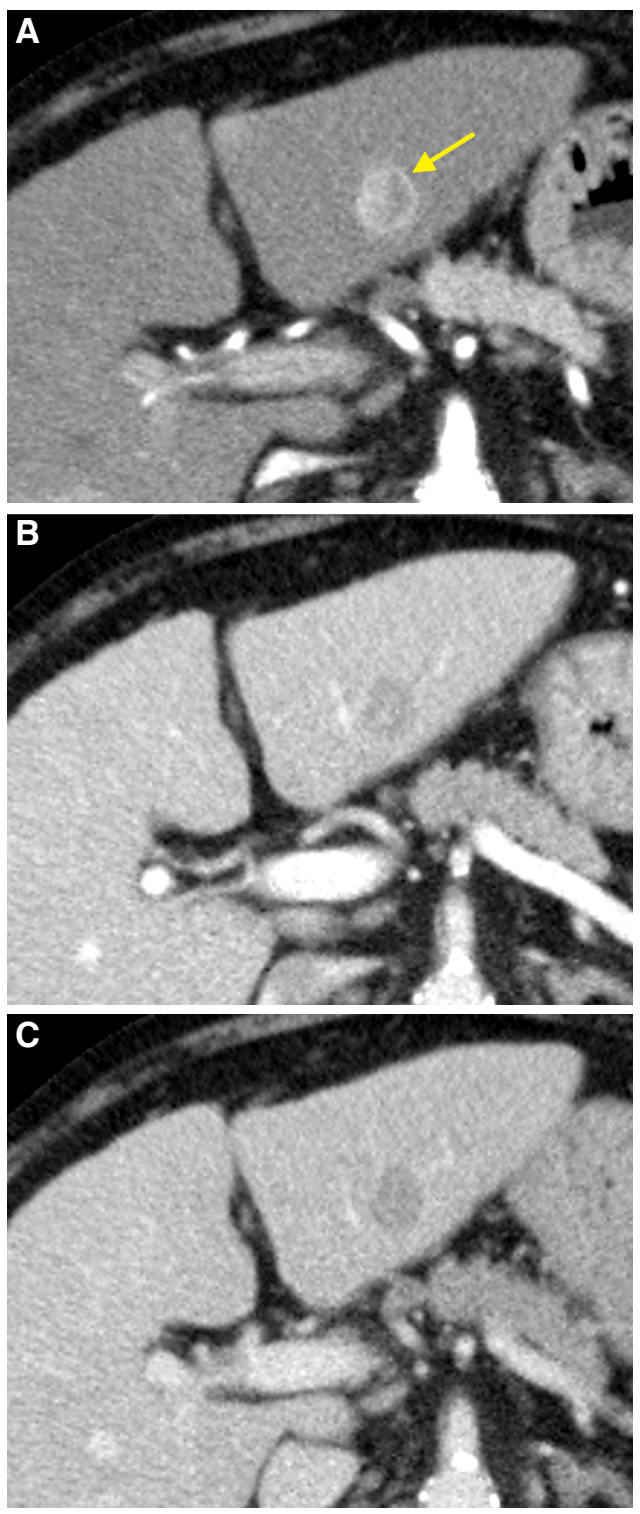

Figure 1: Computed tomography axial planes obtained in arterial phase $(A)$, portal phase $(B)$, and late phase $(C)$. Lesion located in the segment III of left hepatic lobe, heterogeneous enhancement of the lesion is observed in the arterial phase (arrow in A) with washout in the portal and late phases. Mosaic pattern is shown in the arterial and portal phases (yellow arrow) 
Table 1: Magnetic resonance for the study of HCC

\begin{tabular}{l}
\hline Studies \\
\hline FSPGR on phase and opposite phase enhanced on T1 \\
FRFSE enhanced on T2 fat-suppressed \\
LAVA or dynamic 3D SPGRE \\
Pre-contrast phase \\
Post-contrast phase \\
Arterial phase: $16 \mathrm{~s}$ \\
Portal phase: $60 \mathrm{~s}$ \\
Late portal phase: $180 \mathrm{~s}$ \\
Complementary phases: intermediate or later \\
Diffusion \\
B Factor 0 and $600 \mathrm{seg} / \mathrm{mm}^{2}$
\end{tabular}

LAVA: liver acquisition with volume acceleration; HCC: hepatocellular carcinoma

differentiated and poorly differentiated tumors. It has also been shown that atypical enhancement and clearing may even be seen in small $\mathrm{HCC}(<2 \mathrm{~cm}) .{ }^{[6]}$

\section{Magnetic resonance imaging}

MRI is superior to CT in the diagnosis of HCC. The study includes T2 sequences, dual phase-out of phase, dynamic study and diffusion T1 sequences [Table 1].

HCC presents variable signal intensity depending on the degree of fibrosis, necrosis, and fat. It may be hypo, iso, or hyperintense on T1 sequences. On T2 it is generally hyperintense, especially with fat suppression sequences. Gadolinium enhancement shows typical washing as described in CT: enhancement in the arterial phase and typical clearing in portal and late phases [Figure 2]. A mosaic pattern is usually observed.

$\mathrm{MRI}$ is also able to distinguish the fat component of the lesion, which is difficult to detect from CT or US. The capsule of the lesion is hypointense on T1 and may present discrete hyperintensity on T2 with tumor infiltration or edema. In MRI, specific contrasts can be used, especially useful in patients who have not obtained a clear diagnosis by basic imaging. One of the most utilized is gadoxectate disodium. This contrast is taken up by hepatocytes, at approximate rates of $50 \%$, and is then excreted into bile canaliculi, and results in an additional hepatocellular phase of imaging. In this phase, contrast is retained not only by normal liver parenchyma but also by regenerative nodules, dysplastic nodules, and nodular focal hyperplasia. ${ }^{[7]}$ Well differentiated carcinomas may show hyperintensity on hepatobiliary phase; however, most HCC are hypointense. ${ }^{[8]}$

CT and conventional MRI have limitations in detecting small HCC. Hepatobiliary phase provides a more accurate diagnosis in small tumors $(<2 \mathrm{~cm})$, which appear with reduced signal with respect to the surrounding liver, because these tumors do not express the hepatocyte sinusoidal transporter required for uptake. ${ }^{[9]}$

\section{Ultrasonography}

US is a non-invasive test and more accessible. It is possible to determine the size and morphology of the lesion, its location, and possible vascular involvement. It also provides guidance for percutaneous biopsy. Its echogenicity is variable and non-specific and may be hypo- or hyperechoic. The largest lesions are more heterogeneous and often have hypo- or anechoic necrotic areas. With Doppler color, central or peritumoral


Figure 2: Magnetic resonance imaging. Liver acquisition with volume acceleration dynamic sequences obtained in axial planes at $6 \mathrm{~min}(\mathrm{~A}), 9 \mathrm{~min}(\mathrm{~B})$, and $11 \mathrm{~min}(\mathrm{C})$. Enhancement of the lesion (arrow) in early stage $(A)$ and washing (arrow) in the later stages (B and $\mathrm{C}$ ) is observed 



Figure 3: (A) Abdominal ultrasound B-mode showing large heterogeneous mass with hyper- and hypoechoic areas is observed in right hepatic lobe. Peritumoral vascular flow is demonstrated by Doppler (B)

vascular flow can be demonstrated [Figure 3].

According to clinical practice guidelines of the European Association for the Study of the Liver (EASL), ${ }^{[10]}$ a monitoring program must be carried out in patients at high risk for HCC, which mainly includes patients with liver cirrhosis. Abdominal ultrasound is the diagnostic method used and surveillance is conducted every six months. The main limitation of ultrasound is the detection of small tumors (< $2 \mathrm{~cm}$ ). They can go undetected in livers with a heterogeneous diffuse nodular pattern base. However, in expert hands, sensitivity is up to $89 \%$ and specificity is up to $90 \%$.

\section{Contrast-enhanced ultrasound}

Contrast enhanced ultrasound (CEUS) monitors time changes more directly and allows the dynamic study of the lesion. Contrast consists of sulphur hexachloride microbubles of $2.5 \mu \mathrm{m}$ of diameter. Since it is not nephrotoxic and presents few secondary effects, it is useful in patients with nephropathies and in those with known adverse reactions to other contrast agents. CEUS is valuable as a diagnostic tool, as a guide for biopsy and as a measure of treatment response.

Similarly to CT and MRI, CEUS shows a typical vascular pattern in $\mathrm{HCC}$, more frequent in those that are moderately differentiated ${ }^{[11]}$ [Figure 4]. Contrast agent flows exclusively through the intravascular space, without passing to the interstitial liquid, thus explaining some differences with the typical features found in CT or MRI. However, other reports have not found significant differences. Wilson et al. ${ }^{[12]}$ reported no differences in the dynamic behavior among CEUS, MRI and CT. Giorgio et al. ${ }^{[13]}$ did not find any difference between CEUS and CT. Nevertheless, Liu et al. ${ }^{[14]}$ reported different results for small lesions detected by CEUS and CT. In their report, a good correlation was found between both imaging techniques among lesions greater than $2 \mathrm{~cm}$, but there was a low correlation among lesions measuring 1-2 cm. Possible explanations for this discrepancy are the different distribution of contrast agents, the various thickness of the slices of CT, and the effect of the direct time changes measured with CEUS. A cirrhotic background may also cause atypical patterns due to the progressive arterialization of the small lesions. These results suggest that more research is needed to determine the usefulness of CEUS in the diagnosis of HCC.

On the other hand, some papers found that the presence of wash-in/wash-out in CEUS of liver lesions is highly suggestive of cholangiocarcinoma (CC), thus inducing false positive results of HCC. This was observed by Liu et al. ${ }^{[15]}$ in $92.3 \%$ of HCC and in $85.7 \%$ of CC found in 819 patients. However, CC lesions had an earlier washout than HCC lesions (media of 27.5 vs. $70.1 \mathrm{~s}$ ). Up to $68.5 \%$ of CC had a ring enhancement, while it was present in just $2.0 \%$ of HCC. They concluded that an enhancement and washout time longer than $43 \mathrm{~s}$ plus a non-ring enhancement had a $64.1 \%$ sensitivity and a $97.4 \%$ specificity for $\mathrm{HCC}$ lesions equal or smaller than $5 \mathrm{~cm}$.

Ohno et al. ${ }^{[16]}$ observed a linear correlation between blood flow of the lesion and blood flow of the rest of the parenchyma with CEUS in 7 patients, using perflubutane as contrast agent. This activity proves the presence of intratumoral angiogenesis, thus enabling CEUS for measuring response to antiangiogenic therapies, even though the sample size was small in this report.

Nevertheless, the role of CEUS in diagnosis and staging of $\mathrm{HCC}$ is limited and it is not considered a first line diagnostic tool in EASL or American Association for the Study of Liver Diseases (AASLD) guidelines.

CEUS is useful for guiding biopsies. Spârchez et al. ${ }^{[17]}$ 


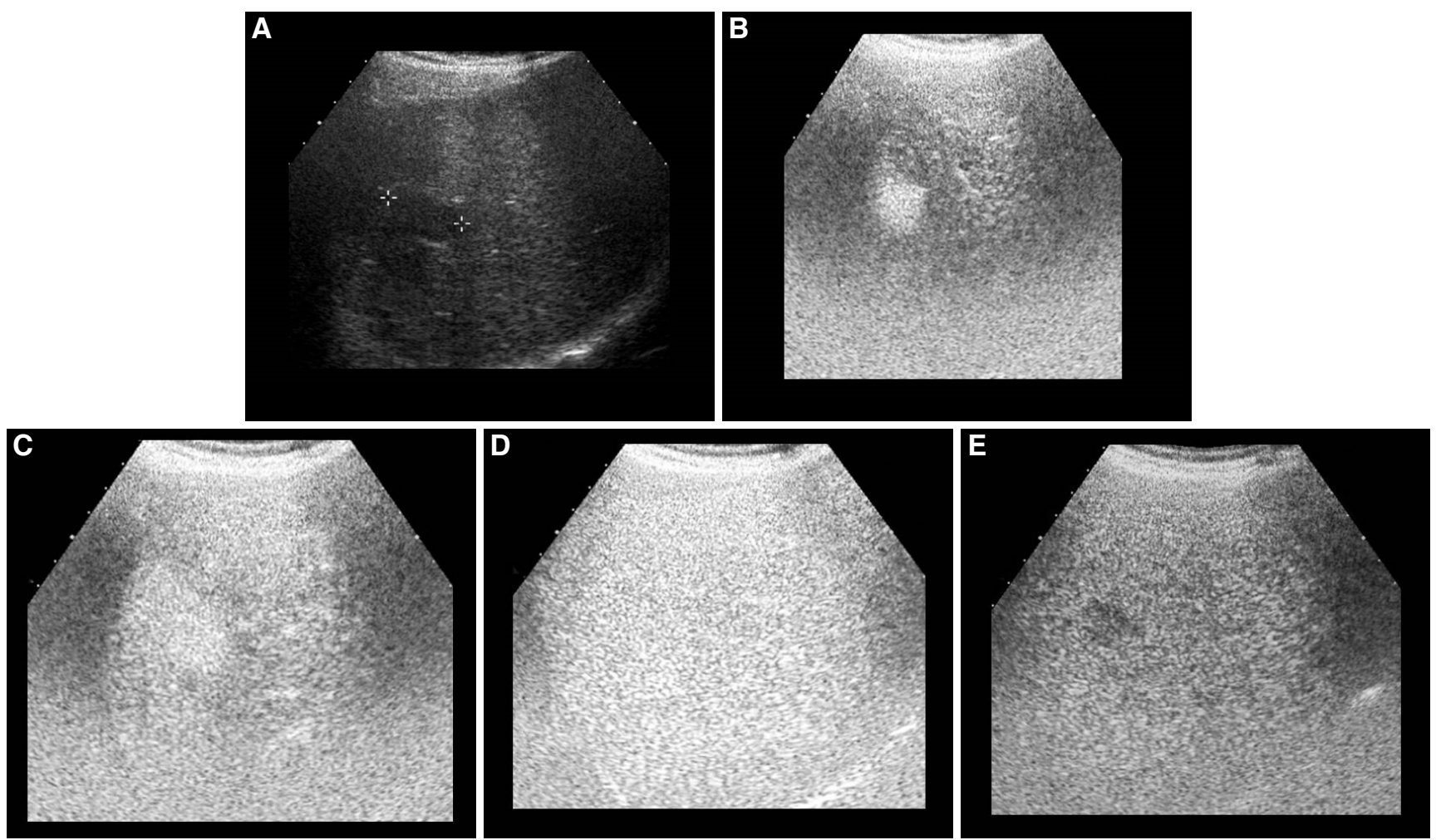

Figure 4: Abdominal ultrasound (A) and with contrast at $23 \mathrm{~s}(\mathrm{~B}), 30 \mathrm{~s}(\mathrm{C}), 1 \mathrm{~min}(\mathrm{D})$, and $5 \mathrm{~min}(\mathrm{E})$. Hypoechoic lesion in right hepatic lobe corresponding to hepatocellular carcinoma with a typical vascular pattern: early uptake in arterial phases (B, C), isoechogenic respect to surrounded liver parenchyma in portal phase (D) and wash-out contrast in late phase (E)

prospectively compared conventional US and CEUS in 171 cirrhotic and non-cirrhotic patients. Biopsy was possible with CEUS in $97.6 \%$ of the cases, obtaining one sole sample in $43.0 \%$ of them, compared with $23.4 \%$ using US. In general, sensitivity was greater with CEUS (96.5\% vs. $81.5 \%)$, also in cirrhotic patients $(95.2 \%$ vs. $75.0 \%)$, in lesions greater than 6 $\mathrm{cm}(97.8 \%$ vs. $82.0 \%)$ and in poorly visualized lesions (100.0\% vs. $66.6 \%)$. When histology was inconclusive with US a new biopsy was performed with CEUS, obtaining a final diagnosis in every case.

CEUS may be useful also to monitor tumor response to treatment. With antiangiogenic therapy, changes in tumoral vascularization precede changes in tumoral size. A complete response may be considered when there is no enhancement at any time. Irregular enhancement and/or eccentrical or peripheral nodules suggest the presence of residual tumor. ${ }^{[18]}$ Using a quantitative analysis an individualized treatment could be done, but more research is needed to establish this indication for CEUS.

CEUS performed 60 min after radiofrequency ablation or alcoholization of HCC may monitor the efficacy of the treatment. ${ }^{[18]}$ Gao et al. ${ }^{[19]}$ measured the different peak enhancement of contrast between tumor and surrounding parenchyma and encountered significantly lower rates in patients with tumor recurrence compared with those without recurrence. On the other hand, the expression levels of basic fibroblast growth factor in the recurrence group were higher than those in the non-recurrence group. Xia et al. ${ }^{[20]}$ and other reports have shown a greater sensitivity of CEUS compared to CT when detecting residual tumor after chemoembolization ( $58.1 \%$ vs. $39.5 \%)$.

CEUS has not shown better sensitivity than CT or MRI when looking for late recurrence. Thus, these two techniques are the gold standard for the long term follow-up of patients with HCC.

\section{MANAGEMENT IN DIAGNOSIS OF HCC}

The objective is early detection. In early stages, radical treatment and improved prognosis are possible. The pathological diagnosis of the tumor involves biopsy of the lesion. It is an invasive technique including risks such as bleeding or tumor seeding.

In 2001, diagnostic criteria for the management of nodular lesions in the cirrhotic liver were established. These criteria favor an early non-invasive diagnosis, preventing biopsy in some cases. In the latest update of the clinical practice guidelines of the EASL (2012), ${ }^{[10]}$ the criteria are as follows: (1) nodules $>2 \mathrm{~cm}$ can be 
diagnosed as $\mathrm{HCC}$ directly with one imaging test with typical findings, if there is early enhancement and late washing; (2) nodules from 1 to $2 \mathrm{~cm}$ require two different techniques for diagnosis with typical findings; and ( 3 ) nodules $<1 \mathrm{~cm}$ should be followed by US every 4 months during the first year and then every 6 months.

In nodules between 1 and $2 \mathrm{~cm}$, the AASLD in its latest update $(2010)^{[21]}$ establish the criteria for a single positive test. However, the EASL does not recommend following this approach in the absence of prospective studies to support it. Both guidelines recommend the use of $C T$ or $M R I$ and limit the use of CEUS. As described in previous sections, intravascular contrast distribution means that in some cases the behavior of the lesion is not typical or obtains false positives in CC. In case of uncertain diagnosis, biopsy of the lesion is required.

\section{RADIOLOGICAL ASSESSMENT OF CIRRHOTIC LIVER COMPARED TO HEALTHY LIVER}

In the assessment by imaging techniques of patients without known cirrhosis three questions must be considered: first, differentiating between healthy and cirrhotic liver; second, determining if there are morphological differences or behavior in HCC that occur in cirrhotic liver versus healthy liver; and third, analyzing the management and differential diagnosis according to these characteristics with other tumors that may be seen in healthy liver, with similar radiological characteristics as $\mathrm{HCC}$.

Chronic liver disease, regardless of its etiology, leads to progressive development of liver fibrosis and then to the final and irreversible stage of cirrhosis. The gross morphological changes that occur in cirrhotic livers are easily detectable with any current imaging techniques. In recent years, new imaging methods, from liver elastography of transition to modern diffusion techniques and MRI elastography, have been developed to assess liver fibrosis with the intention of making a diagnosis at an early stage that allows an active treatment for incipient liver fibrosis. In this article we review the spectrum of chronic liver disease findings in different imaging techniques.

US is usually the first technique used and can detect liver cirrhosis and its complications. In the first phase of cirrhosis liver can be enlarged, whereas in advanced stages the liver is usually small with atrophy of the right lobe (predominantly anterior segment) and the medial segment of the left lobe, and relative enlargement of lateral segments of the left, caudate or both lobes. The morphological patterns of chronic liver disease overlap between the different causes of cirrhosis. However, hypertrophy of the lateral segments, accompanied by atrophy of the right and the left medial lobe segments, occurs frequently in patients with cirrhosis induced by virus. On the other hand, caudate lobe hypertrophy is usually associated with alcoholic cirrhosis. ${ }^{[3]}$ Several studies have evaluated the ratio between the width of the caudate lobe and the right lobe $(C / R L)$ as an indicator of cirrhosis. Awaya et al. ${ }^{[22]}$ considered a value of $C / R L>0.65$ indicative of cirrhosis. The specificity is high (>90\%), but with low sensitivity $(43-80 \%)$, indicating that the quotient $C / R L$ is a useful measure if abnormal. ${ }^{[23-25]}$

Heterogeneous echostructure and multinodular appearance are frequent observations in chronic liver disease. However, its assessment mainly in the initial stages has much variability. ${ }^{[26]}$ The presence of irregular and nodular surface contour of the liver is considered to be a sign of cirrhosis. This alteration is secondary to the presence of fibrosis and regeneration nodes. This sign is easily visible in the presence of ascites, which allows a better evaluation of liver surface through the liquid (88\% sensitivity, $82-95 \%$ specificity). ${ }^{[27]}$ In absence of ascites it is advisable to judge the previous liver surface by high frequency probes $(7.5 \mathrm{MHz})$, increasing the sensitivity in detecting this pattern. Its existence is associated with macronodular cirrhosis.

Fibrosis of liver parenchyma can alter the morphology of the hepatic veins, with alteration in distensibility, causing luminal narrowing because the walls of the hepatic veins are thin. In advanced cases, alteration of venous flow is observed using Doppler-US, with loss of the triphase morphology of the wave flow in the hepatic veins (this condition is called "portalization"). Depending on the degree of fibrosis, intrahepatic arterial branches may be elongated with tortuous appearance with a "corkscrew" morphology, due to the distortion of the underlying liver parenchyma architecture. The wave of the hepatic artery also shows an altered dynamic, with increase of speed secondary to the lower flow of the portal vein.

Another important sign in patients with cirrhosis is detection of portal hypertension. Increased resistance of portal venous blood flow causes increased portal, mesenteric and splenic vein caliber. Thus, the existence of a diameter greater than $13 \mathrm{~mm}$ has a sensitivity of $42 \%$ and a specificity of $90 \%$ for the diagnosis of portal hypertension. ${ }^{[2]}$ The increase of less than $20 \%$ in the diameter of the portal vein with deep inspiration is another sign of portal hypertension, with a sensitivity of $80 \%$ and a specificity of $100 \% .{ }^{[29]}$ However, the difficulty in assessing this measurement 
and the inter-observer variability make this a poor criterion. In cases of severe portal hypertension there may be reversal of flow in the main vein or intrahepatic branches (centrifugal flow), and even thrombosis of the portal vein and portal cavernoma. Other signs of portal hypertension most commonly found in these patients are the presence of ascites, splenomegaly and porto-systemic collaterals (near the gastroesophageal junction, paraumbilical, retroperitoneal, gastro or spleno-renal and hemorrhoidal). However, conventional US does not usually detect abnormalities in liver morphology in patients with mild cirrhosis. The absence of such changes does not exclude this pathology. ${ }^{[30]}$

In the last decade new techniques which quantify the degree of fibrosis have been developed, based on elastography (transient elastography and quantitative elastography) that improve the sensitivity for detection of liver fibrosis. Transient elastography (TE) or FibroScan ${ }^{\circledR}$ is based on the emission of low-frequency elastic waves $(50 \mathrm{~Hz})$ and amplitude through the skin to the target organ. There is an inverse relationship between the speed of wave propagation and tissue elasticity (measured in kilopascals, $\mathrm{kPa}$ ). Thus, there is a higher propagation velocity, with lower tissue elasticity in higher degree of fibrosis. TE has been validated in multiple studies to detect cirrhosis, with a sensitivity of $84-100 \%$ and a specificity of $91-96 \%$. ${ }^{[31]}$

However, TE has low diagnostic efficiency in obese patients, when there is a narrow intercostal space and the presence of ascites, due to poor acoustic window and depth. Quantitative elastography, based on the strength of acoustic radiation impulse (ARFI), is integrated in a conventional US equipment that generates, through the US transducer, an acoustic pulse on the area of interest to evaluate tissue consistency. The transducer produces an US wave drive that causes a longitudinal displacement and determines the appearance of a wave pulse to the longitudinal tangential cut. The speed of the shear wave in the region of interest is directly proportional to the tissue stiffness and is measured in meters/ second. The results are very similar to those achieved with FibroScan ${ }^{\circledR}$. Both techniques show good reliability to identify patients with significant fibrosis (F2) and severe fibrosis (F3), and are excellent for the diagnosis of liver cirrhosis (F4). ${ }^{[32,33]}$

The ARFI system has several advantages compared with TE. With the addition of structural and morphological data to a conventional US, it is a more accurate method of choosing the liver parenchyma fragment to analyze. Also, it avoids structures which distort the results, such as the filling of blood vessels,

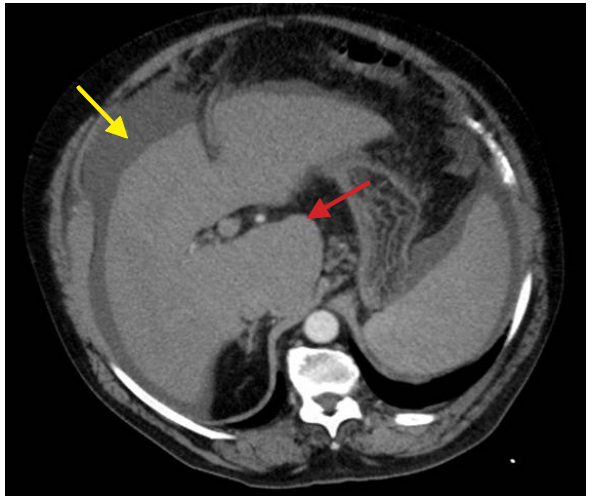

Figure 5: Computed tomography of axial plane in portal phase. Cirrhotic liver: lobed contours (yellow arrow) and moderate hypertrophy of the caudate lobe (red arrow)

gallbladder and ribs or liver capsule. These findings cannot be controlled with the FibroScan $\AA$, since it does not have an associated image. Also, with the ARFI elastography adequate results can be obtained in obese patients with a body mass index $\geq 40 \mathrm{~kg} / \mathrm{m}^{2}$ and even in patients with ascites. ${ }^{[34]}$

The CT is a somewhat sensitive technique for the diagnosis of cirrhosis in its early stage. The contrast used should be preferably of a high iodine concentration $(350-370 \mathrm{mg} / \mathrm{mL})$ and administered at a high injection rate $(4-5 \mathrm{~mL} / \mathrm{s})$. CT findings are similar to those observed by US: contour nodularity, right lobe atrophy, hypertrophy of the left lobe and caudate and increased C/RL index [Figure 5].

In early stages of cirrhosis, hepatic hilum widening is identified in $98 \%$ of the patients in the absence of other typical morphological findings of cirrhosis. However, this finding is also observed in $11 \%$ of patients with healthy liver ${ }^{[34]}$ These patients may also show an increase in size and prominence of the interlobular fissure, with increased extrahepatic fat between the medial segment and left lateral liver secondary to atrophy of the medial hepatic segment. Structural changes in the initial phase cannot be readily assessed.

In advanced stages, heterogeneous attenuation with a diffuse distribution can be seen as well as isodense lesions in the surrounding parenchyma, corresponding to regenerative nodules. Some of them may have an increased basal density due to the presence of iron. In the dynamic study it is possible to detect vascular abnormalities as pseudolesions in the subcapsular location and wedge morphology. They have early focal enhancement, being isodense with the rest of the liver parenchyma in the portal phase. They correspond to small arterioportal shunts that are false positives of HCC, both in CT and MRI. In advanced 
stages of cirrhosis, it is possible to see peripheral hypodense areas, with retraction of the liver contour and delayed enhancement, corresponding to focal confluent fibrosis. Signs of portal hypertension are similar to those seen with US: portal vein dilation, varicose veins and splenomegaly.

MRI shows greater tissue contrast than CT and US, resulting in increased information on the changes in the structure of the liver parenchyma. In patients with advanced cirrhosis, MRI may show a heterogeneous liver parenchyma with regenerative nodules and fibrous septa or bridges. The regenerative nodules are isointense or hyperintense on T1 sequences and isointense or hypointense on T2 sequences. The fibrous septa are crosslinks of low signal intensity on T1 sequences and high intensity on T2.

Areas of confluent focal fibrosis, which appear as hypointense lesions on T1 and hyperintense on T2, can also be identified. Contrast media based on gadolinium are accumulated in the extracellular compartment and are deposited on the fibrous tissue in the liver. Thus, most contrast agents based on gadolinium improve signal of liver fibrosis in T1, particularly in the venous phase and equilibrium phase. It is also possible, as with US, to perform an elastography by MRI, quantifying liver stiffness by analyzing the propagation of mechanical waves through the tissue. It allows assessment of all the liver surface, unlike US elastography, which only evaluates the outermost regions. It has high sensitivity (92\%) and specificity (95\%) for the detection of liver fibrosis. ${ }^{[35]}$ However, it is a technique of limited availability today, with long turnaround times and cannot be done to livers with iron overload due to noise signal artifacts.

Diffusion technique evaluates the diffusion of the protons of water molecules within tissues. It is routinely used for liver testing. Calculating the apparent diffusion coefficient (ADC) can facilitate the assessment of liver fibrosis. It has been shown that ADC values decrease as liver fibrosis increase. Bakan et al. ${ }^{[36]}$ detected no significant differences in ADC values between stages F0 and F1 and between F1 and F2. Another study, however, showed significant differences in ADC values between the stages $\mathrm{F} 0$ and $\mathrm{F} 4 .^{[37]}$ Together, these findings suggest that diffusion technique is not reliable for distinguishing the early stages of liver fibrosis.

Vascular changes that occur as a result of cirrhosis can be detected after the administration of a paramagnetic contrast agent and can be useful to quantify the state of parenchymal microcirculation. Liver fibrosis decreases portal venous flow, increases arterial blood flow and forms intrahepatic shunts. As is the case of diffusion and MRI elastography, perfusion measures the liver fibrosis with indirect markers. Hagiwara et al. ${ }^{[38]}$ showed an increase in absolute blood flow, blood fraction, volume of distribution and the mean transit time, and a decreased portal venous fraction in patients with advanced liver fibrosis compared to patients with early-stage fibrosis. However, several factors may affect the correlation between perfusion parameters and fibrosis (cardiac output, fasting, liver congestion, liver inflammation, liver damage, and portal venous flow).

The study of liver fibrosis by molecular MRI is still in its development phase and is emerging as a valuable tool for the non-invasive detection of early-stage liver fibrosis. Compared to normal liver, the amount of type I collagen in fibrotic livers increases significantly (from $36 \%$ to $53 \%$ ). ${ }^{[39]}$ Therefore, type I collagen can be used as a molecular target for detection of liver fibrosis by molecular MRI. Research on the development of specific radiopharmaceuticals which can target only the extracellular matrix collagen for the diagnosis of early-stage fibrotic livers is underway.

From the above it is concluded that US, CT, and conventional MRI have a high specificity for the diagnosis of cirrhosis, but have a low sensitivity in the early stages of the disease. In pre-cirrhotic patients, the liver parenchyma usually appears normal on MRI or only a mild non-specific heterogeneity of the parenchyma is identified. Using discrete elastography can improve the sensitivity in detecting early cirrhosis. Göbel et al. ${ }^{[40]}$ showed a $10 \%$ increase in sensitivity for detection of liver cirrhosis with TE compared to the use of routine screening. They also showed that the combination of TE with conventional US further improves diagnostic accuracy. However, at present, with current imaging techniques, the absence of fibrosis or cirrhosis in patients with lesions suspicious of HCC cannot be confirmed.

Liver biopsy is considered the gold standard for evaluating fibrosis. ${ }^{[41]}$ However, it is an invasive procedure which can be associated with pain and with a $0.5 \%$ risk of complications. ${ }^{[42]}$ Moreover, this technique has limitations: first, biopsy analyzes a small part of the parenchyma, leading to sampling errors if it has been done in an area with less fibrotic component; second, there is a $20 \%$ intra- and inter-observer variability in the histological assessment: ${ }^{[43]}$ and third, it should be noted that the biopsy does not predict disease progression and therefore additional biopsies would be needed after starting treatment for follow-up.

In the absence of morphological signs of cirrhosis in patients with suspicious lesions of $\mathrm{HCC}$, histological 

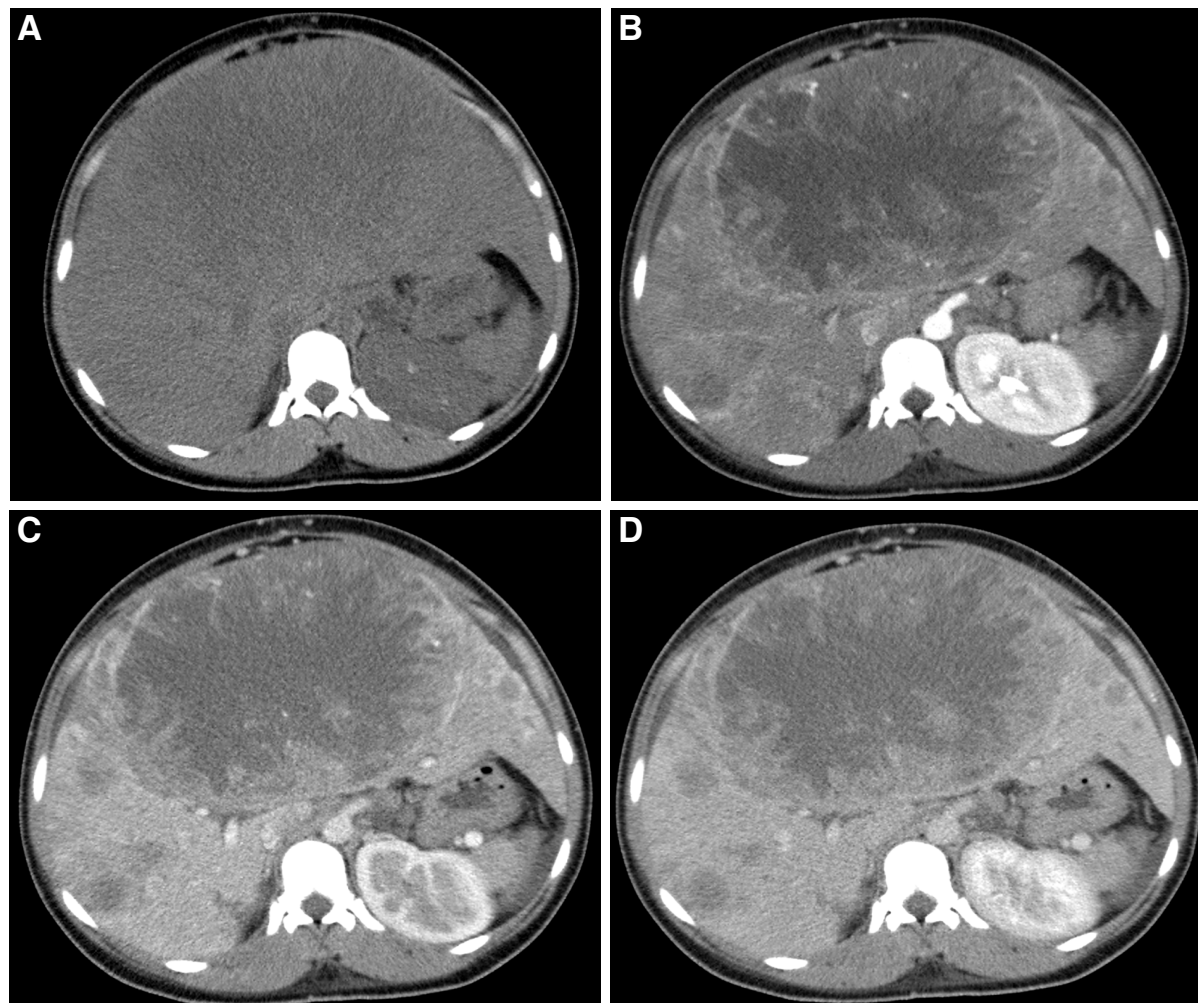

Figure 6: Computed tomography obtained in axial planes (A) and arterial phase (B), portal phase (C), and late phase (D). Voluminous mass, encapsulated, with extensive necrosis and presence of multiple satellite lesions is identified in non-cirrhotic liver. The mass shows peripheral enhancement, predominantly in the arterial phase (B) and no contrast washout are observed in later phases (C, D). The findings are compatible with hepatocellular carcinoma with atypical behaviour

assessment of hepatic parenchyma is a controversial choice. Di Martino et al. ${ }^{[4]}$ demonstrated that noninvasive diagnostic criteria of HCC are present in $90 \%$ of cases and that the HCC in non-cirrhotic patients shows a similar pattern of enhancement as HCC in cirrhotic patients. Based on these results it would be reasonable to apply non-invasive diagnostic criteria for HCC in non-cirrhotic patients if they have high levels of $\alpha$-fetoprotein.

\section{DIFFERENCES OF PRESENTATION OF HCC IN CIRRHOTIC VS. NON-CIRRHOTIC LIVERS}

Ninety percent of HCC arise mainly in a liver with established cirrhosis resulting from chronic $\mathrm{HCV}$ or $\mathrm{HBV}$ infection or alcohol related liver disease. ${ }^{[45]}$

Radiologists are used to see the imaging of HCC that arises in cirrhotic livers. In these cases, the tumor is often multifocal or diffuse and small in relation to the screening area visualized in these patients. $\mathrm{HCC}$ in non-cirrhotic liver is an uncommon finding for radiologists, presenting with different clinical and treatment options as well as prognosis. ${ }^{[4,46]}$

The setting of HCC in non-cirrhotic liver is twice more common in men than in women, but there is a lower prevalence of male presentation regarding $\mathrm{HCC}$ in cirrhotic liver. The average patient age at diagnosis is 65 years old. ${ }^{[3]}$ There is little literature on the radiological characteristics of this tumor in non-cirrhotic liver. Winston et al. ${ }^{[47]}$ described the characteristics of MRI in 25 patients with HCC in noncirrhotic liver, compared with 11 patients with HCC in cirrhotic liver. In the group of non-cirrhotic patients, HCC usually presents as large masses (with an average size of $12.4 \mathrm{~cm}$ ), predominantly solitary or dominant with small satellite lesions ( $82 \%$ of patients) [Figure 6]. In patients with cirrhosis, tumors are generally smaller. Their larger size and extent at time of diagnosis in non-cirrhotic livers could be explained by the non-inclusion of these patients in prevention programs. In healthy livers, there is a predisposition for HCC to occur in the right hepatic lobe. ${ }^{[4]}$

The usually well-differentiated HCC is an encapsulated tumor with circumscribed margins, while poorly differentiated HCC is an aggressive tumor that is not encapsulated and has an ill-defined outline [Figure 7]. These findings are more prevalent in HCC in cirrhotic liver whereas the HCC in non-cirrhotic liver is predominantly moderate or well differentiated. ${ }^{[49]}$ This lesion may contain calcifications, necrosis, haemorrhage, and microscopic and macroscopic fat [Figure 8]. Sometimes, 



Figure 7: Computed tomography of axial planes in the arterial phase (A) and portal phase (B). Mass in the caudate lobe (arrows), non-capsulated, is identified in non-cirrhotic liver. Lesion presents heterogeneous enhancement in arterial phase $(A)$ and late wash-out $(B)$

there may be focal dilatation of intrahepatic bile duct [Figure 9]; this finding is secondary to the mass effect produced by these tumors, as already mentioned, and they may reach a large size.

A greater tendency of extrahepatic spread, by direct invasion of adjacent structures or by distant spread as metastasis $\left(20.5 \%\right.$ vs. $6.5 \%$, respectively) ${ }^{[4]}$ has been documented for HCC in non-cirrhotic livers compared to cirrhotic livers. This difference can be explained by a delayed diagnosis.


Figure 8: Computed tomography of axial planes in empty $(A)$ and portal phase (B). Liver mass in non-cirrhotic liver with calcifications (black arrow) and important vascular component (white arrow)

In non-cirrhotic patients, $\mathrm{HCC}$ has a similar radiologic behavior as in cirrhotic patients. On US, HCC usually appears as a hypoechoic or more often hyperechoic non-specific lesion. In larger size lesions, a heterogeneous echostructure should be observed, due to combining solid and necrotic areas.

In CT studies without contrast the tumor tends to be hypodense relative to the surrounding liver parenchyma. Calcifications can be identified as well as areas of necrosis and hemorrhage. Following administration of intravenous contrast, the tumor typically shows
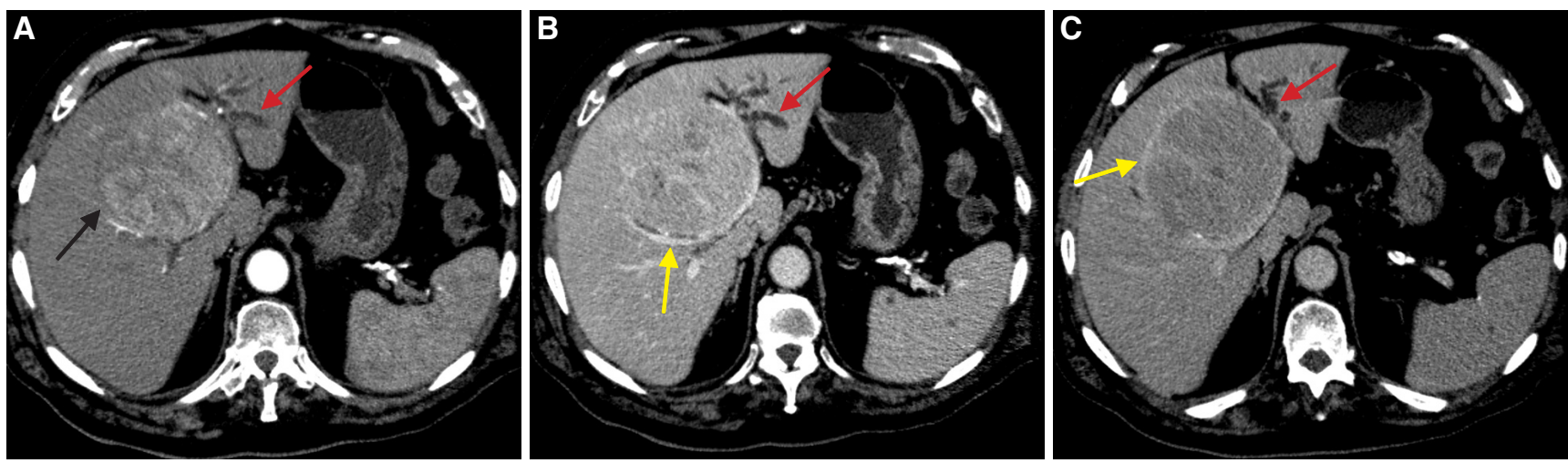

Figure 9: Computed tomography of axial planes obtained in arterial phase (A), portal phase (B), and late phase (C). Non-cirrhotic liver shows mass in right hepatic lobe (black arrow) with typical behavior of hepatocellular carcinoma. Heterogeneous enhancement in the arterial phase (A), and portal phase (B) with wash-out in delayed phase (C). The mass shows enhanced capsule in late phase (yellow arrow) and produces secondary dilatation of the bile duct (red arrow) 

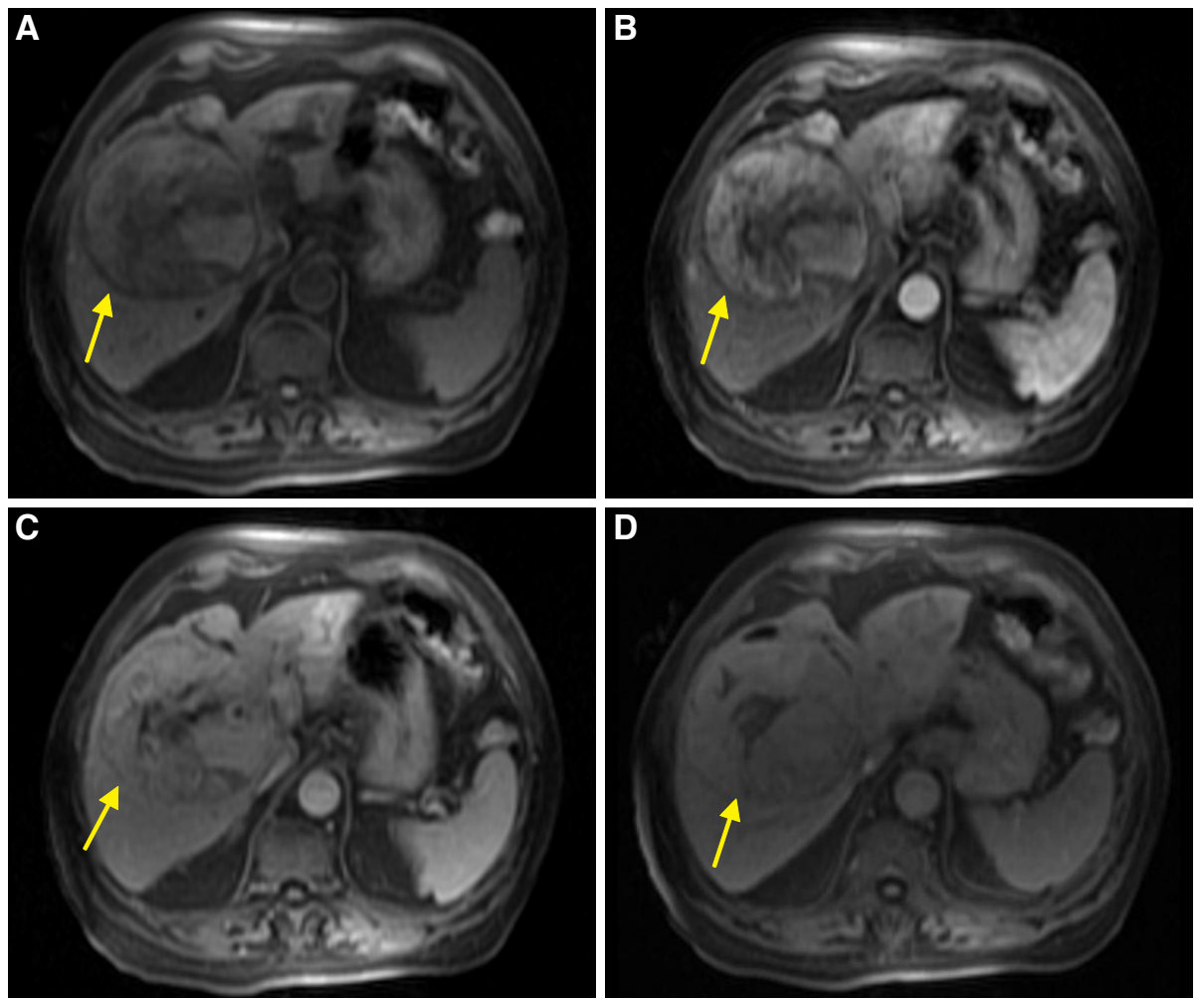

Figure 10: Magnetic resonance imaging of liver acquisition with volume acceleration dynamic sequences in axial planes: empty ( $A$ ), arterial phase (B), portal phase (C), and delayed phase (D). Non-cirrhotic liver shows mass in the right hepatic lobe (yellow arrows) with necrotic component that presents heterogeneous enhancement in the arterial phase (B), and wash-out in portal phase (C) and delayed phase (D). These findings are compatible with hepatocellular carcinoma with typical behaviour

enhancement during the arterial phase (wash-in), becoming isodense in the early portal phase, and wash-out in the late portal phase and equilibrium with respect to the adjacent liver parenchyma, similar to the HCC in the cirrhotic liver [Figure 9]. Capsular enhancement, when present, is most apparent during the equilibrium phase.

The appearance of HCC on MRI in healthy liver also has the same radiological features as that in cirrhotic liver. On T1 sequences it will be most commonly hypointense relative to the surrounding liver parenchyma, although it may contain hyperintense areas due to the presence of hemorrhage and fat within the lesion. Microscopic fat can be seen in about $10-17 \%$ of non-cirrhotic HCC, similar to HCC in cirrhotic livers. It is a finding most often seen in welldifferentiated tumors and, therefore, a sign of good prognosis. On T2 sequences, the HCC will be usually isointense or hyperintense. However, well or poorly differentiated tumors can be isointense or hypointense. In dynamic sequences after gadolinium administration, they will show a typical pattern identical to the enhancement on CT [Figure 10].

Usually, there will be an internal enhancement mosaic, also described in previous sections, which become clearer mainly in the post-contrast study. It may be surrounded by a capsule with a similar behavior: hypointense on T1 and hyperintense on post-contrast study. In $80 \%$ of cases there may be a pseudocapsule formed by prominent peritumoral vessels or fibrosis, where iodinated contrast and gadolinium may be retained, producing a circumferential enhancement in the late portal phase or equilibrium phase.

In a retrospective review of 209 patients with diagnosis of HCC in our center over a period of 4 years (January 2010 - December 2014), 23 patients were selected with healthy liver by histological criteria (liver biopsy or surgical resection piece) and/or a combination of clinical, analytical criteria, imaging and hepatic hemodynamics. The average age at diagnosis in these patients was 70 years old, with no significant differences in distribution by sex, as opposed to the higher incidence in males described by other authors. ${ }^{[3]}$ Most diagnostic testing was initiated by the presence of abdominal pain or abnormal liver profiles, as in other studies. ${ }^{[50]}$ Twenty-one patients were diagnosed with $\mathrm{HCC}$ by biopsy and/or surgery.

Congruent with previous studies, the presentation of HCC was as a single large lesion $(65 \%)$ or a dominant mass with satellite lesions (35\%), with a 

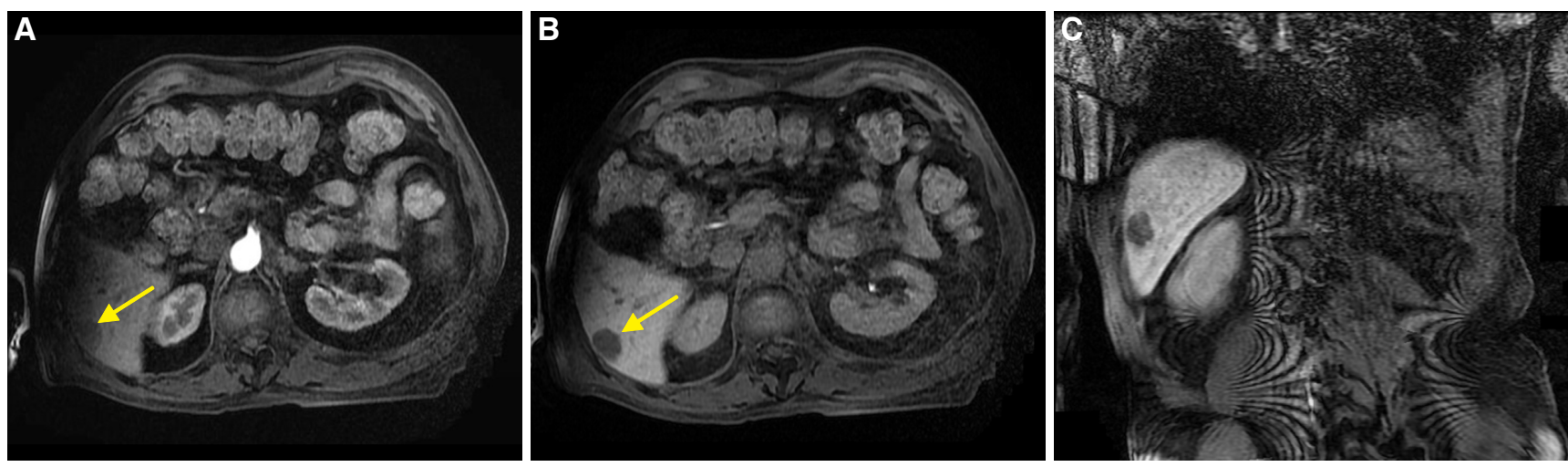

Figure 11: Magnetic resonance imaging in dynamic sequences: axial in arterial phase (A), 10 min (B), and coronal plane at 20 min (C). Image A, B, and C show a non-cirrhotic liver with focal lesion (yellow arrows) in segment VI. Lesion is hypovascular in all phases and present atypical behavior for hepatocellular carcinoma

largest mean diameter of $10.7 \mathrm{~cm}$. The right lobe was the most common location (57\%). The presence of capsule $(60 \%)$, well-circumscribed margin $(70 \%)$, intratumoral necrosis $(87 \%)$ and a typical behavior $(60 \%)$ in the dynamic study after administration of intravenous contrast were present in the radiological characteristics in most HCC. Five patients $(22 \%)$ had distant metastases and $3(13 \%)$ patients had portal vein thrombosis.

\section{DIFFERENTIAL DIAGNOSIS WITH OTHER ENTITIES IN THE CONTEXT OF NON- CIRRHOTIC LIVER}

The role of biopsy in the diagnosis of HCC is controversial. Tumor spread after biopsy is unusual, but recent meta-analysis has reported an overall prevalence of $2.7 \%$ and an annual rate of $0.9 \%$ after performing biopsy. ${ }^{[51]}$ The AASLD and EASL advocate different guidelines for the diagnosis of HCC using specific imaging criteria. ${ }^{[52]}$ Biopsy is limited to lesions $>1 \mathrm{~cm}$ with indeterminate characteristics in two image techniques. There is no guideline regarding the management of HCC in non-cirrhotic patients compared to that in cirrhotic patients. ${ }^{[44]}$ However, a lesion with imaging characteristics of HCC in these patients without increased serum levels of alphafetoprotein, in a non-endemic area of HCC, makes it necessary to rule out other tumors. Therefore, in these cases performing a biopsy may be recommended.

There are several hypervascular lesions similar to HCC. So, faced with a hypervascular lesion detected with any imaging technique, it is necessary to make a differential diagnosis between several entities such as focal nodular hyperplasia (FNH), hepatocellular adenoma (HA) or other malignancies such as intrahepatic cholangiocarcinoma (ICC), primary neuroendocrine tumors of the liver and hypervascular liver metastases. Moreover, atypical HCC may present as a hypovascular lesion [Figure 11] or with other characteristics.

FNH [Figure 12] is formed by benign-appearing hyperplastic hepatocytes in normal liver stroma. The typical US appearance is a nodule isoechoic with the normal liver parenchyma. A central scar, containing dense connective tissue and thick arteries, is present in $77 \%$ of the cases. This scar appears usually as a hypoechoic area with a central artery that presents low resistance flow in Doppler study. In CT without contrast it is usually seen as a well-defined isodense or slightly hypodense mass compared to liver parenchyma. The scar is hypodense. Following intravenous contrast administration, in the arterial phase there is a homogeneous and intense uptake, with the central scar remaining hypodense. Later, progressive washout makes it isodense in portal and late phases. The central scar, on the contrary, shows a progressive uptake being hypodense or isodense in portal phase and hyperdense in late phase.

MRI may be useful in the characterization of the lesion in order to identify the central scar in a higher number of cases. In both sequences, T1 and T2, $\mathrm{FNH}$ may be difficult to distinguish from normal liver parenchyma remaining as an isointense or slightly hypointense mass on T1 and hyperintense on T2. The behavior in the dynamic contrast is similar to CT. Due to the hepatocellular origin of the lesion, when contrast with hepatobiliary elimination is used, the uptake of the lesion remains isointense or slightly hyperintense relative to normal parenchyma, due to increased secretion and excretion of contrast material of the lesion with respect to the remaining liver parenchyma. The key to the differential diagnosis with $\mathrm{HCC}$ is the presence of a similar enhancement of liver parenchyma in portal and delayed phases after contrast administration and the retention of hepatoespecific contrast. 



Figure 12: Computed tomography: axial planes obtained in arterial phase (A) and portal phase (B). Focal lesion in left hepatic lobe (yellow arrow) shows enhancement in the arterial phase $(A)$ and is isodense in the portal phase (B) with central scar (red arrow). Magnetic resonance imaging: liver acquisition with volume acceleration dynamic sequences in axial planes: noncontrast phase (C), arterial phase (D), hepatocyte phase (E) and portal phase (F). Focal lesion is hypointense in noncontrast phase (C) with enhancement in arterial (D) and hepatocyte phases $(\mathrm{E})$, with central scar. Lesion is isointense in delayed phase $(\mathrm{F})$. Lesion shows typical radiological findings of focal nodular hyperplasia

Table 2: Magnetic resonance imaging differentiation between the three subtypes of hepatocellular adenoma

\begin{tabular}{|c|c|c|c|c|}
\hline Type & T1 & T1FF & T2 & $\mathrm{T} 1+\mathrm{C}$ \\
\hline Inflammatory adenoma & $\begin{array}{l}\text { Moderately hyperintense or } \\
\text { isointense }\end{array}$ & No signal drop & $\begin{array}{l}\text { Hyperintense, greater } \\
\text { peripheral intensity }\end{array}$ & $\begin{array}{l}\text { Enhancement in arterial phase and persists } \\
\text { in portal phase and late phase }\end{array}$ \\
\hline $\begin{array}{l}\text { Mutated HNF1A } \\
\text { adenoma }\end{array}$ & Hyperintense or isointense & Hypointense & Isointense & $\begin{array}{l}\text { Enhancement in arterial phase that does } \\
\text { not persist in portal phase and late phase }\end{array}$ \\
\hline $\begin{array}{l}\text { Mutated beta-catenin } \\
\text { adenoma }\end{array}$ & Non specific pattern & $\begin{array}{l}\text { Non specific } \\
\text { pattern }\end{array}$ & Non specific pattern & $\begin{array}{l}\text { Similar to hepatocellular carcinoma: } \\
\text { enhancement in arterial phase and } \\
\text { washing in portal phase and late phase }\end{array}$ \\
\hline
\end{tabular}

$\mathrm{HA}$ is a rare benign tumor. It is currently classified into 4 subgroups depending on their genotype: inflammatory adenoma, mutated HNF1A adenoma, mutated beta-catenin hepatocellular adenoma and unrated. They show different clinical behavior so their management is different. ${ }^{[53]}$

HA are hypervascular and heterogeneous lesions caused by foci of bleeding and may contain fat. Using Doppler color, intra-lesional flow can be identified, unlike $\mathrm{FNH}$ or $\mathrm{HCC}$, and it does not produce a pulsatile continuous curve. In CT they are well-defined lesions, hypodense to isodense or slightly hyperdense with respect to the parenchyma. They may have a heterogeneous density and/or areas of hemorrhage. In contrast CT they are hypervascular and show significant enhancement in the arterial phase. In portal and late phases they differ by subtype: inflammatory adenoma shows a persistent enhancement, mutated HNF1A adenoma is isodense regarding the parenchyma, mutated beta-catenin adenoma appears hypervascular in the arterial phase and washes the contrast like HCC [Figure 13].

MRI is the technique of choice for the differentiation of the three subtypes, with the features shown in Table 2. Inflammatory adenoma is the most common subtype. Histologically it is composed of inflammatory infiltrate and dilation of sinusoids. It is the subtype with the higher risk of bleeding. Mutated beta-catenin adenoma is the least common subtype but that which presents the greater risk of malignant transformation 



Figure 13: Computed tomography of axial planes obtained in empty (A), arterial phase (B), and portal phase (C). It shows healthy liver with hypodense mass in empty (A) with intense enhancement in the arterial phase (B), and washed-out in portal phase (C). The first radiological and pathological diagnosis was hepatic adenoma. A second biopsy confirmed the diagnosis of hepatocellular carcinoma

$(5-10 \%)$. It is more common in men with deposition diseases or who consume anabolic steroids.

HCC should be distinguished from ICC with mass growth pattern. Although they are malignant tumors, prognosis and treatment are very different in both entities. The typical enhancement pattern of ICC is a gradual contrast uptake without washing $(80 \%$ of ICC) or stable contrast uptake without washing $(20 \%$ of ICC). In arterial phase, it appears as a hypodense mass with incomplete peripheral enhancement. The central part shows a prolonged enhancement in the late phase, due to the slowness of washing related to the fibrous tissue in the tumor [Figure 14]. The pattern of progressive or stable enhancement in portal and late phases can also sometimes be observed in HCC. Therefore, with this type of pattern we always perform a biopsy for histological diagnosis.

Liver can also be a frequent site of metastatic neuroendocrine tumors from another location [Figure 15]; it is unlikely to be of primary liver origin. The primary hepatic carcinoid tumor appears as a liver mass, usually solid $(60 \%)$, partially solid with cystic areas
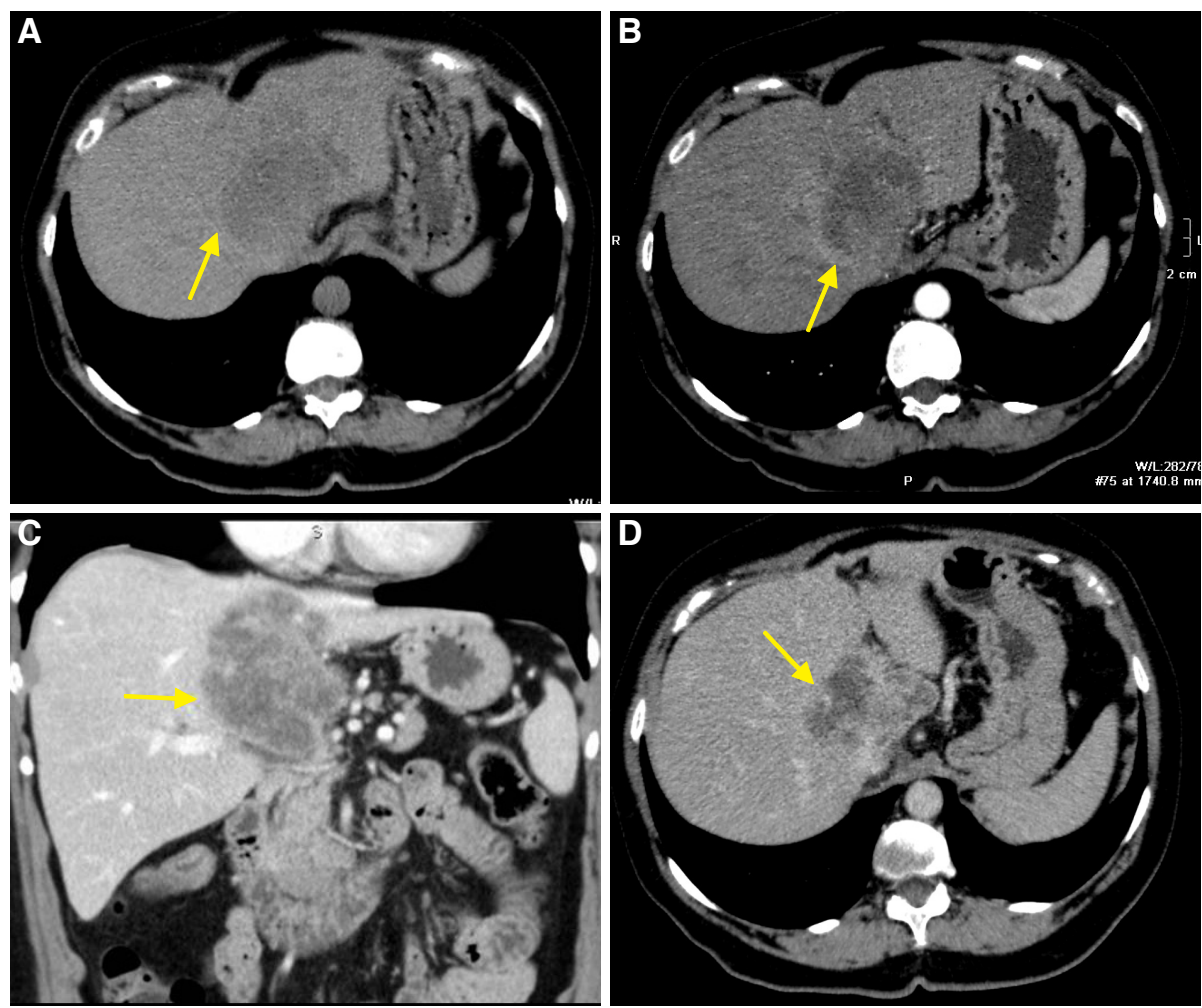

Figure 14: Computed tomography of axial planes obtained in noncontrast phase $(A)$, arterial phase $(B)$, portal phase in coronal plane $(C)$ and axial plane in delayed phase (D). Hepatic mass (yellow arrow) hypodense in noncontrast phase (A), with heterogeneous peripheral enhancement in the arterial phase (B), and portal phase (C), and central uptake in delayed phase (D). This lesion corresponded to cholangiocarcinoma 

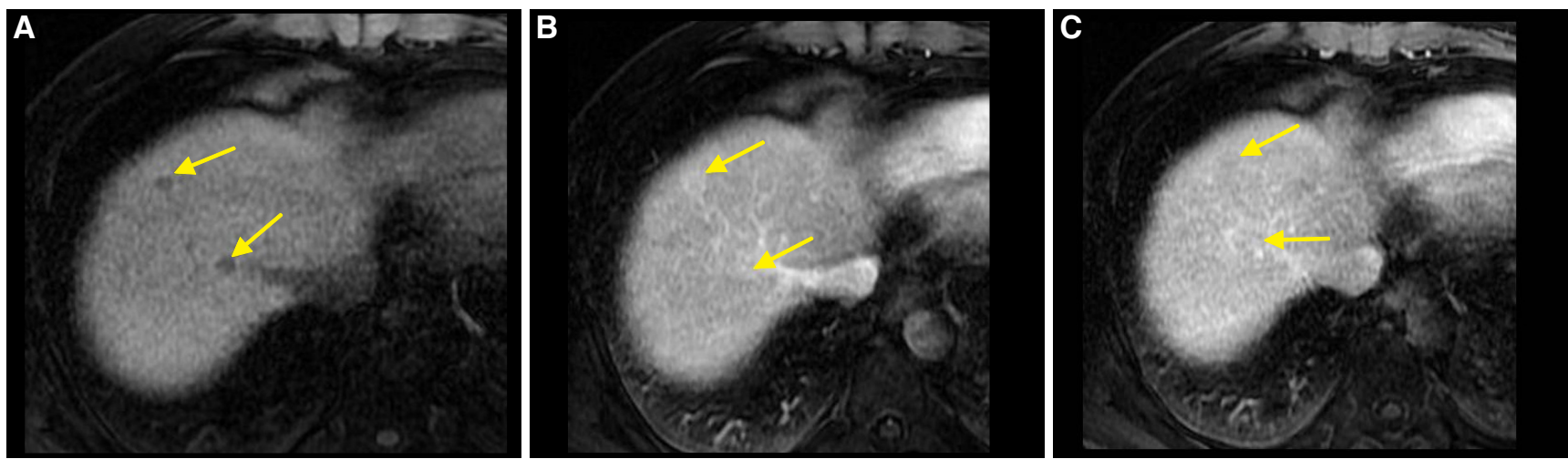

Figure 15: Magnetic resonance imaging in axial planes with T1 sequences in noncontrast phase (A), and arterial contrast phase (B), and portal phase (C). Multiple lesions (yellow arrows) scattered in right hepatic lobe, hypointense in noncontrast phase (A) and uptake in early arterial phase $(B)$, held in portal phase $(C)$ corresponding to liver metastases of pancreatic neuroendocrine tumor

$(25 \%)$, or predominantly cystic $(15 \%)$. It shows peripheral enhancement in the arterial phase. In MRI it is hypointense on T1 and hyperintense on T2 and with an enhancement after administration of gadolinium similar to that obtained in CT.

In addition to the metastatic neuroendocrine tumors, other tumors with hypervascular appearance such as thyroid tumors, renal tumors or melanomas, may present as an initial liver finding. Such lesions are generally multiple and small, unlike the usual presentation of HCC. The uptake curve of hypervascular metastases is typical: very intense and early enhancement in the arterial phase and also very early wash-out in the portal and equilibrium phases. This dynamic behavior is similar to that presented in HCC and therefore, if a primary tumor is not known and there is a small number of lesions, biopsy is essential for the differential diagnosis.

In the absence of typical signs of benign lesion as $\mathrm{FNH}$ or inflammatory adenoma and with a suspicion of malignancy, a reliable diagnosis cannot be made and a histologic confirmation is required due to the similarity of the radiologic features of these lesions with typical HCC.

In conclusion, $\mathrm{HCC}$ in patients with healthy livers have no significant differences in dynamics and morphological characteristics. However, they are usually diagnosed in more advanced phases and are larger, probably because they are not subjected to screening programs. Due to the similar properties of other benign or malignant lesions, the diagnosis must be made by biopsy unlike in cirrhotic patients, where a lesion with early and late enhancement washing (wash-in and wash-out) is pathognomonic of HCC and a biopsy is not needed. On the other hand, a cirrhotic substrate cannot be ruled out by imaging techniques. Therefore, in the absence of other clinical and laboratory data suggesting a history of cirrhosis, biopsy should be performed in all lesions with pathognomonic characteristics of HCC.

\section{Financial support and sponsorship None.}

\section{Conflicts of interest}

There are no conflicts of interest.

\section{Patient consent}

There is no patient involved.

\section{Ethics approval}

This review paper is waived for ethics approval.

\section{REFERENCES}

1. Ferlay J, Soerjomataram I, Dikshit R, Eser S, Mathers C, Rebelo M, Parkin DM, Forman D, Bray F. Cancer incidence and mortality worldwide: sources, methods and major patterns in GLOBOCAN 2012. Int J Cancer 2015;136:E359-86.

2. SEER Cancer Statistics Factsheets: Liver and Intrahepatic Bile Duct. Available from: http://seer.cancer.gov/statfacts/html/livibd.html. [Last accessed on Aug 29 2016]

3. Gaddikeri S, McNeeley MF, Wang CL, Bhargava P, Dighe MK, Yeh MM, Dubinsky TJ, Kolokythas O, Lalwani N. Hepatocellular carcinoma in the noncirrhotic liver. AJR Am J Roentgenol 2014;203:W34-47.

4. Trevisani F, D’Intino PE, Caraceni P, Pizzo M, Stefanini GF, Mazziotti A, Grazi GL, Gozzetti G, Gasbarrini G, Bernardi M. Etiologic factors and clinical presentation of hepatocellular carcinoma. Differences between cirrhotic and noncirrhotic Italian patients. Cancer 1995; 75:2220-32.

5. Lee JH, Lee JM, Kim SJ, Baek JH, Yun SH, Kim KW, Han JK, Choi BI. Enhancement patterns of hepatocellular carcinomas on multiphasicmultidetector row CT: comparison with pathological differentiation. Br J Radiol 2012;85:e573-83,

6. Iavarone M, Sangiovanni A, Forzenigo LV, Massironi S, Fraquelli M, Aghemo A, Ronchi G, Biondetti P, Roncalli M, Colombo M. Diagnosis of hepatocellular carcinoma in cirrhosis by dynamic contrast imaging: the importance of tumor cell differentiation. Hepatology 
2010;52:1723-30.

7. Seale MK, Catalano OA, Saini S, Hahn PF, Sahani DV. Hepatobiliaryspecific MR contrast agents: role in imaging the liver and biliary tree. Radiographics 2009;29:1725-48.

8. Kim MJ, Rhee HJ, Jeong HT. Hyperintense lesions on gadoxetate disodium-enhanced hepatobiliary phase imaging. AJR Am J Roentgenol 2012;199:W575-86.

9. Tsuboyama T, Onishi H, Kim T, Akita H, Hori M, Tatsumi M, Nakamoto A, Nagano H, Matsuura N, Wakasa K, Tomoda K. Hepatocellular carcinoma: hepatocyte-selective enhancement at gadoxetic acid-enhanced MR imaging--correlation with expression of sinusoidal and canalicular transporters and bile accumulation. Radiology 2010;255:824-33.

10. European Association For The Study Of The Liver; European Organisation For Research And Treatment Of Cancer. EASL-EORTC clinical practice guidelines: management of hepatocellular carcinoma. J Hepatol 2012;56:908-43.

11. Jang HJ, Kim TK, Burns PN, Wilson SR. Enhancement patterns of hepatocellular carcinoma at contrast-enhanced US: comparison with histologic differentiation. Radiology 2007;244:898-906.

12. Wilson SR, Kim TK, Jang HJ, Burns PN. Enhancement patterns of focal liver masses: discordance between contrast-enhanced sonography and contrast-enhanced CT and MRI. AJR Am J Roentgenol 2007;189:W7-12.

13. Giorgio A, Ferraioli G, Tarantino L, de Stefano G, Scala V, Scarano F, Coppola C, Del Viscovo L. Contrast-enhanced sonographic appearance of hepatocellular carcinoma in patients with cirrhosis: comparison with contrast-enhanced helical CT appearance. AJR Am J Roentgenol 2004; 183:1319-26.

14. Liu JJ, Li HX, Chen ZB, Yang WP, Zhao SF, Chen J, Bai T, Li H, Li LQ. Consistency analysis of contrast-enhanced ultrasound and contrast-enhanced CT in diagnosis of small hepatocellular carcinoma. Int J Clin Exp Med 2015;8:21466-71.

15. Liu GJ, Wang W, Lu MD, Xie XY, Xu HX, Xu ZF, Chen LD, Wang Z, Liang JY, Huang Y, Li W, Liu JY. Contrast-enhanced ultrasound for the characterization of hepatocellular carcinoma and intrahepatic cholangiocarcinoma. Liver Cancer 2015;4:241-52.

16. Ohno N, Miyati T, Yamashita M, Narikawa M. Quantitative assessment of tissue perfusion in hepatocellular carcinoma using perflubutane dynamic contrast-enhanced ultrasonography: a preliminary study. Diagnostics 2015;5:210-8.

17. Spârchez Z, Radu P, Kacso G, Spârchez M, Zaharia T, Al Hajjar N. Prospective comparison between real time contrast enhanced and conventional ultrasound guidance in percutaneous biopsies of liver tumors. Med Ultrason 2015;17:456-63.

18. Roccarina D, Garcovich M, Ainora ME, Riccardi L, Pompili M, Gasbarrini A, Zocco MA. Usefulness of contrast enhanced ultrasound in monitoring therapeutic response after hepatocellular carcinoma treatment. World J Hepatol 2015;7:1866-74.

19. Gao Y, Zheng DY, Cui Z, Ma Y, Liu YZ, Zhang W. Predictive value of quantitative contrast-enhanced ultrasound in hepatocellular carcinoma recurrence after ablation. World J Gastroenterol 2015;21:10418-26.

20. Xia Y, Kudo M, Minami Y, Hatanaka K, Ueshima K, Chung H, Hagiwara S, Inoue T, Ishikawa E, Kitai S, Takahashi S, Tatsumi C, Ueda T, Hayaishi S, Maekawa K: Response evaluation of transcatheter arterial chemoembolization in hepatocellular carcinomas: the usefulness of sonazoid-enhanced harmonic sonography. Oncology 2008;75:99-105.

21. Bruix J, Sherman M; American Association for the Study of Liver Diseases. Management of hepatocellular carcinoma: an update. Hepatology 2011;53:1020-2.

22. Awaya H, Mitchell DG, Kamishima T, Holland G, Ito K, Matsumoto T.
Cirrhosis: modified caudate-right lobe ratio. Radiology 2002;224:76974.

23. Harbin WP, Robert NJ, Ferrucci JT. Diagnosis of cirrhosis based on regional changes in hepatic morphology: a radiological and pathological analysis. Radiology 1980;135:273-83.

24. Giorgio A, Amoroso P, Lettieri G, Fico P, de Stefano G, Finelli L, Scala V, Tarantino L, Pierri P, Pesce G. Cirrhosis: value of caudate to right lobe ratio in diagnosis with US. Radiology 1986;161:443-5.

25. Hess CF, Schmiedl U, Koelbel G, Knecht R, Kurtz B. Diagnosis of liver cirrhosis with US: receiver-operating characteristic analysis of multidimensional caudate lobe indexes. Radiology 1989;171:349-51.

26. Sanford NL, Walsh P, Matis C, Baddeley H, Powell LW. Is ultrasonography useful in the assessment of diffuse parenchymal liver disease? Gastroenterology 1985;89:186-91.

27. Bluth E, Arger P, Benson C, Ralls P, Siegel M. Ultrasound, a practical approach to clinical problems. New York: Thieme Publishing Group; 2008. p.36.

28. McGahan J, Goldberg B. Diagnostic ultrasound. New York: Informa Healthcare; 2008.

29. Robinson KA, Middleton WD, Al-Sukaiti R, Teefey SA, Dahiya N. Doppler sonography of portal hypertension. Ultrasound $Q$ 2009;25:313.

30. Lin DY, Sheen IS, Chiu CT, Lin SM, Kuo YC, Liaw YF Ultrasonographic changes of early liver cirrhosis in chronic hepatitis B: a longitudinal study. J Clin Ultrasound 1993;21:303-8.

31. Afdhal NH. Fibroscan (transient elastography) for the measurement of liver fibrosis. Gastroenterol Hepatol (N Y) 2012;8:605-7.

32. Ganne-Carrié N, Ziol M, de Ledinghen V, Douvin C, Marcellin P, Castera L, Dhumeaux D, Trinchet JC, Beaugrand M. Accuracy of liver stiffness measurement for the diagnosis of cirrhosis in patients with chronic liver diseases. Hepatology 2006;44:1511-7.

33. Sporea I, Badea R, Sirli R, Lupsor M, Popescu A, Danila M, Focsa M, Deleanu A. How efficient is acoustic radiation force impulse elastography for the evaluation of liver stiffness? Hepat Mon 2011;11:532-8.

34. Ito K, Mitchell DG, Gabata T. Enlargement of hilar periportal space: a sign of early cirrhosis at MR imaging. J Magn Reson Imaging 2000;11:136-40

35. Wang Y, Ganger DR, Levitsky J, Sternick LA, McCarthy RJ, Chen ZE, Fasanati CW, Bolster B, Shah S, Zuehlsdorff S, Omary RA, Ehman RL, Miller FH. Assessment of chronic hepatitis and fibrosis: comparison of MR elastography and diffusion-weighted imaging. AJR Am J Roentgenol 2011;196:553-61.

36. Bakan AA, Inci E, Bakan S, Gokturk S, Cimilli T. Utility of diffusionweighted imaging in the evaluation of liver fibrosis. Eur Radiol 2012;22:682-7.

37. Sandrasegaran K, Akisik FM, Lin C, Tahir B, Rajan J, Saxena R, Aisen AM. Value of diffusion-weighted MRI for assessing liver fibrosis and cirrhosis. AJR Am J Roentgenol 2009;193:1556-60.

38. Hagiwara M, Rusinek H, Lee VS, Losada M, Bannan MA, Krinsky GA, Taouli B. Advanced liver fibrosis: diagnosis with 3D whole-liver perfusion MR imaging--initial experience. Radiology 2008;246:92634.

39. Gressner AM, Weiskirchen R. Modern pathogenetic concepts of liver fibrosis suggest stellate cells and TGF-beta as major players and therapeutic targets. J Cell Mol Med 2006;10:76-99.

40. Göbel T, Schadewaldt-Tümmers J, Greiner L, Poremba C, Häussinger D, Erhardt A. Transient elastography improves detection of liver cirrhosis compared to routine screening tests. World J Gastroenterol 2015;21:953-60

41. Afdhal NH, Nunes D. Evaluation of liver fibrosis: a concise review. Am J Gastroenterol 2004;99:1160-74.

42. Macarini L, Stoppino L. Practical management of chronic viral 
hepatitis. Chapter 5. Radiologic assessment of liver fibrosis. Present and future. Rijeka, Croatia: InTech; 2013. p.371.

43. Standish RA, Cholongitas E, Dhillon A, Burroughs AK, Dhillon AP. An appraisal of the histopathological assessment of liver fibrosis. Gut 2006;55:569-78.

44. Di Martino M, Saba L, Bosco S, Rossi M, Miles KA, Di Miscio R, Lombardo CV, Tamponi E, Piga M, Catalano C. Hepatocellular carcinoma (HCC) in non-cirrhotic liver: clinical, radiological and pathological findings. Eur Radiol 2014;24:1446-54.

45. Bruix J, Sherman M; Practice Guidelines Committee, American Association for the Study of Liver Diseases. Management of hepatocellular carcinoma. Hepatology 2005;42:1208-36.

46. Lubrano J, Huet E, Tsilividis B, François A, Goria O, Riachi G, Scotté M. Long-term outcome of liver resection for hepatocellular carcinoma in noncirrhotic nonfibrotic liver with no viral hepatitis or alcohol abuse. World J Surg 2008;32:104-9.

47. Winston CB, Schwartz LH, Fong Y, Blumgart LH, Panicek DM. Hepatocellular carcinoma: MR imaging findings in cirrhotic livers and noncirrhotic livers. Radiology 1999;210:75-9.

48. McLarney JK, Rucker PT, Bender GN, Goodman ZD, Kashitani N,
Ros PR. Fibrolamellar carcinoma of the liver: radiologic-pathologic correlation. Radiographics 1999;19:453-71.

49. Nzeako UC, Goodman ZD, Ishak KG. Hepatocellular carcinoma in cirrhotic and noncirrhotic livers. A clinico-histopathologic study of 804 North American patients. Am J Clin Pathol 1996;105:65-75.

50. Brancatelli G, Federle MP, Grazioli L, Carr BI. Hepatocellular carcinoma in noncirrhotic liver: CT, clinical, and pathologic findings in 39 U.S. residents. Radiology 2002;222:89-94.

51. Silva MA, Hegab B, Hyde C, Guo B, Buckels JA, Mirza DF. Needle track seeding following biopsy of liver lesions in the diagnosis of hepatocellular cancer: a systematic review and meta-analysis. Gut 2008;57:1592-6.

52. Mitchell DG, Bruix J, Sherman M, Sirlin CB. LI-RADS (Liver Imaging Reporting and Data System): summary, discussion, and consensus of the LI-RADS Management Working Group and future directions. Hepatology 2015;61:1056-65.

53. Kim H, Jang JJ, Kim DS, Yeom BW, Won NH. Clinicopathological analysis of hepatocellular adenoma according to new bordeaux classification: report of eight korean cases. Korean J Pathol 2013;47:411-7. 